\title{
The Forcing Restrained Steiner Number of a Graph
}

\author{
M. S. Malchijah Raj, J. John
}

\begin{abstract}
A restrained Steiner set of a connected graph $G$ of order $p \geq 2$ is a set $W \subseteq V(G)$ such that $W$ is a Steiner set, and if either $W=V$ or the subgraph $G[V-W]$ inducedby $[V-W]$ has no isolated vertices. The restrained Steiner number $s_{r}(G)$ of $G$ isthe minimum cardinality of its restrained Steiner sets and any restrained Steinerset of cardinality $s_{r}(G)$ is a minimum restrained Steiner set of $G$. For a minimum restrained Steiner set Wof $G$, a subset $T \subseteq W$ is called a forcing subset for $W$ if $W$ is the unique minimum restrained Steiner set containing T. A forcing subset for Wof minimum cardinality is a minimum forcing subset of $W$. The forcing restrained Steiner number of $W$, denoted by $f_{r s}(W)$, is the cardinality of a minimum forcingsubset of $W$. The forcing restrained Steiner number of $G$, denoted by $\left.f_{r s}(G) i s f_{r s}(G)=\min f_{r s}(W)\right\}, \quad$ where the minimum is taken over all minimum restrainedSteiner sets $W$ in G. Some general properties satisfied by the concept forcing restrained Steiner number are studied. The forcing restrained Steiner number of certain classes of graphs is determined. It is shown that for every pair $a, b$ ofintegers with $0 \leq a<$ band $b \geq 2$, there exists a connected graph $G$ such that $f_{r s}(G)=a$ and $s_{r}(G)=b$.

Keywords:
\end{abstract}

Steiner distance, Steiner number, forcing Steiner number, restrained, Steiner number, forcing restrained Steiner number.

\section{INTRODUCTION}

By a graph $G=(V, E)$, we mean a finite undirected graph without loops or multiple edges. The order and size of $G$ are denoted by $p$ and $q$ respectively.The distance $d(u, v)$ between two vertices $u$ and $v$ in a connected graph $G$ is the length of a shortest $u v$ path in $G$. A $u-v$ path of length $d(u, v)$ is called an $u-v$ geodesic. It is known that the distance is a metric on the vertex set $V(G)$. For basic graph theoretic terminology we refer to $[6,1]$. For a vertex $v$ of $G$, the eccentricitye $(v)$ is the distance between $v$ and a vertex farthest from $v$. The minimum eccentricity among the vertices of $G$ is the radius, $\operatorname{rad} G$ and the maximum eccentricity is its diameter, diamG of $G$. Two vertices $x$ and $y$ are antipodalif $d(x, y)=\operatorname{diamG}$. A vertex $v$ is an extreme vertex of a graph $G$ if the subgraph induced by its neighbors is complete. If $e=\{u, v\}$ is an edge of a graph $G$ with $d(u)=1$ and $d(v)>1$, then we call $e$ a pendant edge, $u$ a leaf or end vertex and $v$ a support.A geodetic set of $G$ is a set $S \subseteq V(G)$ such that every vertex of $G$ iscontained in a geodesic joining some pair of vertices in $S$.The geodetic number $g(G)$ of $G$ is the minimum cardinality of its geodetic sets and any geodetic set of cardinality $g(G)$ is a minimum geodetic set.

Revised Manuscript Received on 20 September, 2019.

M. S. Malchijah Raj, Scholar, Research and Development Centre, Bharathiar University, Coimbatore - 641 046, INDIA.

J. John, Department of Mathematics, Government College of Engineering, Tirunelveli- 627 007, INDIA.
For a nonempty set $W$ of vertices in a connected graph $G$, the Steiner distance $d(W)$ of $W$ is the minimum size of a connected subgraph of $G$ containing $W$. Necessarily, eachsubgraph is a tree and is called a Steiner tree with respect to $W$ or a Steiner $W$ - tree. Itis to be noted that $d(W)=d(u, v)$, when $W=\{u, v\}$. If $v$ is an end vertex of a Steiner $W$ - tree, then $v \in W$. Also if $\langle W\rangle$ is connected, then any Steiner $W$ - tree containsthe elements of $W$ only. $S(W)$ denotes the set of all vertices that lie on Steiner $W$ trees.A set $W \subseteq V(G)$ is called a Steiner set of $G$ if every vertex of $G$ lies on some Steiner $W$ - tree or if $S(W)=$ $V(G)$. A Steiner set of minimum cardinality is a minimum Steinerset or simply a $S$ - set and this cardinality is the Steiner number $s(G)$ of $G$. The Steinernumber of a graph was introduced in [4]. The Steiner number of a graph was furtherstudied in $[5,7,11]$.Steiner tree problem is a distance related invariant that arises by considering the cheapest subgraph that connects a given set of vertices. It has applications in design of computer circuits, long distance telephone lines, or mail routing, combinatorial optimization,etc. It is also used to construct roads of minimum total length to interconnect severalhighways. The computational nature of the problem makes it a traditional research subject in theory of computing. They have numerous application in industries as well. A setW of vertices of a graph $G$ is a restrained Steiner set if $W$ is a Steiner set, and if either $W=V$ or the $\operatorname{subgraph} G[V-W]$ induced by $V-W$ has no isolated vertices. Theminimum cardinality of a restrained Steiner set of $G$ is the restrained Steiner number of $G$, and is denoted by $S_{r}(G)$. A restrained Steiner set of minimum cardinality is calledthe $s_{r}$ - set of $G$. This concept is introduced and studied in [8]. The forcing Steinernumber of a graph is introduced in [13] and further studied in [14]. The forcing conceptis applied in various graph parameters by several authors. In this paper we study theforcing concept in restrained Steiner set of a connected graph.

The following theorems are used in the sequel.

Theorem 1.1. [8] Each extreme vertex of a graph $G$ belongs to every restrained Steinerset of $G$.

Theorem 1.2. [8] Let $G$ be a non trivial tree which is not a star. Then $s_{r}(G)$ is equalto the set of all end vertices of $G$.

Theorem 1.3. [8]For any tree $T$ with $p \geq 3$ vertices, $s_{r}(T)=$ pif and only if $T$ is astar.

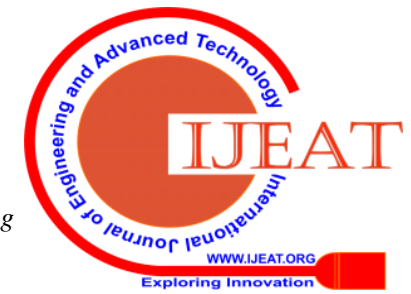




\section{The Forcing Restrained Steiner Number of a Graph}

Throughout the following $G$ denotes a connected graph with at least two vertices.

\section{THE FORCING RESTRAINED STEINER NUMBER OF A GRAPH}

Even though every connected graph contains a minimum restrained Steiner set, some connected graph may contain several minimum restrained Steiner sets. For each minimum restrained Steiner set $W$ in a connected graph $G$, there is always some subset $T$ of $W$ that uniquely determines $W$ as the minimum restrained Steiner set containing $T$. We consider such "forcing subsets" in this section.

Definition 2.1Let $G$ be a connected graph and $W$ a restrained Steiner set of $G$. Asubset $T \subseteq W$ is called a forcing subset for $W$ if $W$ is the unique minimum restrainedSteiner set containing T. A forcing subset for $W$ of minimum cardinality is a minimumforcing subset of $W$. The forcing restrained Steiner number of $W$, denoted by $f_{r s}(W)$, isthe cardinality of a minimum forcing subset of $W$. The forcing restrained Steiner numberof $G$, denoted by $f_{r S}(G)$ is $\left.f_{r s}(G)=\operatorname{minff}_{r s}(W)\right\}$, where the minimum is taken over allminimum restrained Steiner sets $W$ in $G$.

Example 2.2. For the graph $G$ given in Figure 2.1, $W=$ $\left\{v_{1}, v_{3}\right\}$ is the unique minimumrestrained Steiner set of $G$ so that $f_{r s}(G)=0$. For the graph $G$ given in Figure 2.2, $W_{1}=$ $\left\{v_{1}, v_{3}, v_{6}\right\}$ and $W_{2}=\left\{v_{1}, v_{4}, v_{6}\right\}$ are the only two $s_{r}$ - sets of $G$. It is clear that $f_{r s}\left(W_{1}\right)=1$ and $f_{r s}\left(W_{2}\right)=1$ so that $f_{r s}(G)=1$

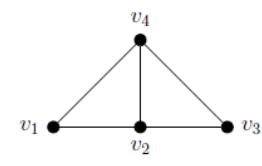

$G$ Figure 2.1

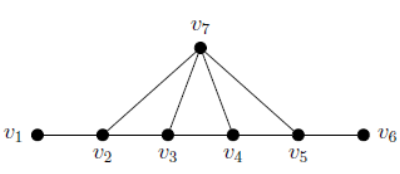

G

Figure 2.2
The next Theorem follows immediately from the definition of the restrained Steinernumber and the forcing restrained Steiner number of a connected graph $G$.

Theorem 2.3.For every connected graph $G, 0 \leq f_{r s}(G) \leq$ $s_{r}(G)$.

Remark 2.4. The bounds in Theorem 2.3 are sharp. For the graph $G$ given in Figure2.1, $f_{r s}(G)=0$. Also, all the inequalities in the theorem are strict. For the graph $G$ given in Figure 2.2, $f_{r s}(G)=1$ and $s_{r}(G)=3$ so that $0<$ $f_{r s}(G)<s_{r}(G)$.

Theorem 2.5. Let $G$ be a connected graph. Then

a) $f_{r s}(G)=0$ if and only if $G$ has a unique minimum restrained Steiner set.

b) $f_{r s}(G)=1$ if and only if $G$ has at least two minimum restrained Steiner sets, one

of which is a unique minimum restrained Steiner set containing one of its elements, and

c) $f_{r s}(G)=s_{r}(G)$ if and only if no minimum restrained Steiner set of $G$ is the unique minimum restrained Steiner set containing any of its proper subsets.

Definition 2.6. A vertex $v$ of a connected graph $G$ is a restrained Steiner vertex of $G$ if $v$ belongs to every restrained Steiner set of $G$. If $G$ has a unique restrained Steiner set $W$, then every vertex of $W$ is a restrained Steiner vertex of $G$.

Example 2.7. For the graph $G$ given in Figure 2.2, $W_{1}=$ $\left\{v_{1}, v_{3}, v_{6}\right\}$ and $W_{2}=\left\{v_{1}, v_{4}, v_{6}\right\}$ are the only two $s_{r}$ - sets of $G$ so that every $s_{r}$ - set contains the vertices $v_{1}$ and $v_{6}$. Hence the vertices $v_{1}$ and $v_{6}$ are the restrained Steiner vertices of $G$.

Theorem 2.8. Let $G$ be a connected graph and $S$ be the set of all restrained Steinervertices of $G$. Then $f_{r_{S}}(G) \leq s_{r}(G)-$ $|S|$.

Proof. Let $W$ be a minimum restrained Steiner set of $G$. Then $s_{r}(G)=|W|$. It is clearthat $S \subseteq W$. Let $T$ be the unique minimum forcing restrained Steiner set containing $W-S$. Thus $f_{r s}(G) \leq|W-S| \leq|W|-|S|=$ $s_{r}(G)-|S|$.

Corollary 2.9.If $G$ is a connected graph with $k$ extreme vertices, then $f_{r s}(G) \leq s_{r}(G)-k$.

Proof. The proof follows from Theorems 1.1 and 2.8.

Remark 2.10. The bound in Theorem 2.8 is sharp. For the graph $G$ given in Figure2.2, $W_{1}=\left\{v_{1}, v_{3}, v_{6}\right\}$ and $W_{2}=$ $\left\{v_{1}, v_{4}, v_{6}\right\}$ are the only two $s_{r}$ - sets of $G$ such that $f_{r s}\left(W_{1}\right)=$ 1 and $f_{r s}\left(W_{2}\right)=1$ so that $f_{r s}(G)=1$ and $s_{r}(G)=3$. Also, $S=\left\{v_{1}, v_{6}\right\}$ is theset of all restrained Steiner vertices of $G$ and so $f_{r s}(G)=s_{r}(G)-|S|$. Also, the inequalityin Theorem 2.8 can be strict. For the graph $G$ given in Figure 2.3, $W_{1}=\left\{v_{1}, v_{4}, v_{7}\right\}$ and $W_{2}=\left\{v_{1}, v_{5}, v_{8}\right\}$ are the only two $s_{r}$ - sets of $G$ so that $s_{r}(G)=3$. It is clear that $f_{r s}\left(W_{1}\right)=$ $f_{r s}\left(W_{2}\right)=1$ so that $f_{r s}(G)=1$. Now, $v_{1}$ is the only restrained Steiner vertex of $G$ and so $|S|=1$. Thus $f_{r s}(G)<$ $s_{r}(G)-|S|$.

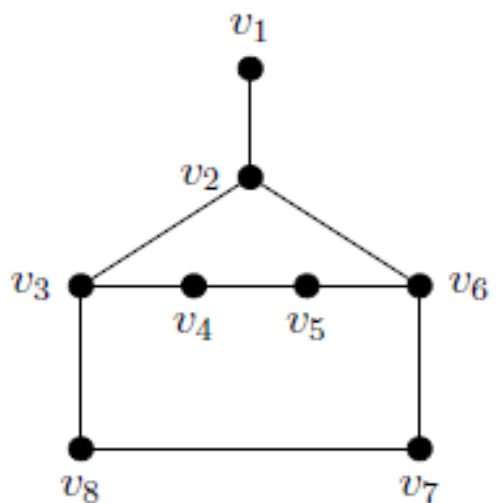

$G$

Figure 2.3 
In the following we determine the forcing restrained Steiner number of some standardgraphs.

Theorem 2.11.For the complete graph $K_{p}(p \geq$
3, frs $K p=0$.

Proof. Since the set of all vertices of a complete graph $K_{p}$ is the unique minimum restrained Steiner set, it follows from Theorem 2.5(a) that $f_{r s}\left(K_{p}\right)=0$.

Theorem 2.12.For the complete bipartite graph $K_{m, n}(m, n \geq 2), f_{r s}\left(K_{m, n}\right)=0$.

Proof. For the complete bipartite graph $K_{m, n}(m, n \geq 2)$, we have $s_{r}\left(K_{m, n}\right)=m+n$. Itfollows from Theorem 2.5(a) that $f_{r s}\left(K_{m, n}\right)=0$.

Theorem 2.13. Let $G$ be a non trivial tree. Then $f_{r s}(G)=0$.

Proof.If $G$ is not a star, then by Theorem 1.2, the set of all end vertices of $G$ is theunique minimum restrained Steiner set of $G$. Now, it follows from Theorem 2.5(a) that $f_{r s}(G)=$ 0 .

If $G$ is a star $K_{1, p-1}$, then it follows from Theorem 1.3, that the set of all vertices of $G$ is the unique minimum restrained Steiner set of $G$. By Theorem 2.5(a) it follows that $f_{r s}(G)=$ 0 .

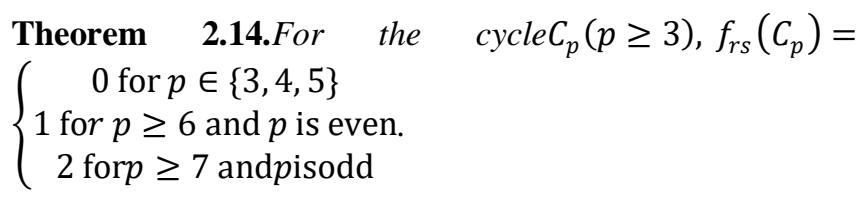

Proof. It is proved in [8] that the restrained Steiner number $s_{r}\left(C_{p}\right)=p$ for $p \in\{3,4,5\}$ and so it follows from Theorem 2.5(a) that $f_{r s}\left(C_{p}\right)=0$ for $p \in\{3,4,5\}$. Let $p \geq 6$. Foran even cycle $C_{p}$, the restrained Steiner set consists of a pair of antipodal vertices of $C_{p}$ and from Theorem $2.5(\mathrm{~b})$ it follows that $f_{r s}\left(C_{p}\right)=1$. Let $p$ be odd with $p=2 n+1, n=1,2,3, \ldots$.

Let the cycle be $C_{p}: v_{1}, v_{2}, \ldots \ldots, v_{n}, v_{n+1}, v_{n+2}, \ldots \ldots, v_{2 n+1}, v_{1}$. If $W=\{u, v\}$ isany set of two vertices of $C_{p}$, then no vertex of the $u-v$ longest path lies on the Steiner $W$ - tree in $C_{p}$ and so no two element subset of $C_{p}$ is a Steiner set of $C_{p}$. Now it is clearthat the sets $W_{1}=\left\{v_{1}, v_{n+1}, v_{n+2}\right\}, W_{2}=\left\{v_{2}, v_{n+2}, v_{n+3}\right\}, \ldots ., W_{2 n+1}=$ $\left\{v_{2 n+1}, v_{n}, v_{n+1}\right\}$ are the $s$ - sets of $C_{p}$. Since $p \geq 7$, the $\operatorname{subgraph} G\left[V-W_{i}\right](1 \leq i \leq 2 n+1)$ has no isolatedvertices. Therefore the $s$ - sets of $C_{p}$ are the $s_{r}$ - sets of $C_{p}$. It is clear from the $s_{r}-\operatorname{sets} W_{i}(1 \leq i \leq 2 n+1)$ that each $\left\{v_{i}\right\}(1 \leq i \leq 2 n+1)$ is a subset of more than one $s_{r}-$ set $W_{i}$. Hence it follows from Theorem 2.5(a) and (b) that $f_{r s}\left(C_{p}\right) \geq 2$. Now since $v_{n+1}$ and $v_{n+2}$ are antipodal to $v_{1}$, it is clear that $W_{1}$ is the unique $s_{r^{-}}$set containing $\left\{v_{n+1}, v_{n+2}\right\}$ and so $f_{r s}\left(C_{p}\right)=2$. Hence the proof.

Theorem 2.15.For the wheel $W_{p}=K_{1}+C_{p-1},(p \geq$ 5, $s r W p=p-3$ and frs $W p=p-4$.
Proof. . Let $G=W_{p}$. Let $v$ be the vertex of $K_{1}$ and let $v_{1}, v_{2}, \ldots ., v_{p-1}, v_{1}$ be the cycle $C_{p-1}$. For $p=5$, let $W_{1}=\left\{v_{1}, v_{3}\right\}$ and $W_{2}=\left\{v_{2}, v_{4}\right\}$. Then $W_{i}, i=1,2$ is a Steiner set of $G$. Since the $\left.\operatorname{subgraph} G\left[V-W_{i}\right], i=1,2\right]$ has no isolated vertices, $W_{i}, i=1,2$ is a restrained Steiner set of $G$ so that $s_{r}\left(W_{p}\right)=2=p-3$ and $f_{r s}\left(W_{p}\right)=1=$ $p-4$. Let $p \geq 6$. Let $W$ be a Steiner set of $G$. If $v \in W$, then $\langle W\rangle$ is connected. Then the Steiner $W$ - tree contains elements of $W$ only. Therefore $v \notin W$. Hence $W \subseteq V\left(C_{p-1}\right)$. Let $W$ be any subset of vertices of $C_{p-1}$ of cardinality $p-$ 3obtained by deleting two non-adjacent vertices of $C_{p-1}$. We may assume without lossof generality that $W=$ $\left\{v_{1}, v_{2}, \ldots, v_{i-1}, v_{i+1}, v_{i+2}, \ldots, v_{j-1}, v_{j+1}, v_{j+2}, \ldots, v_{p-1}\right\}$,

where $1 \leq i<j \leq p-1$ and $j \geq i+2$. Since the subgraph induced by $G[V-W]$ has noisolated vertices, it is clear that $W$ is a minimun restrained Steiner set of $G$ so that $s_{r}\left(W_{p}\right) \leq$ $|W|=p-3$. Let $W^{\prime}$ be any restrained Steiner set with $\left|W^{\prime}\right| \leq p-4$ Thenat least three vertices of $C_{p-1}$ do not belong to $W^{\prime}$. If these vertices are consecutiveon $C_{p-1}$, then $W^{\prime}$ is not a Steiner set of $G$. Otherwise there are non adjacent vertices $v_{k}$ and $v_{l}(k<l)$ such that $v_{k}, v_{l} \notin W^{\prime}$. Since the size of any Steiner $W^{\prime}$ - tree is atmost $p-4$, it is easily seen that $v_{k}$ and $v_{l}$ do not lie on any Steiner $W^{\prime}$ - tree of $G$ andso $W^{\prime}$ is not a restrained Steiner set of $G$. Thus $s_{r}\left(W_{p}\right)=p-3$. Since the subgraphinduced by a proper Steiner set is disconnected, it follows that any $s_{r}$ - set is of the

form $W=$

$\left\{v_{1}, v_{2}, \ldots, v_{i-1}, v_{i+1}, v_{i+2}, \ldots, v_{j-1}, v_{j+1}, v_{j+2}, \ldots, v_{p-1}\right\}$

where $v_{i}$ and $v_{j}$ arenon-adjacent. Let $T$ be a subset of $W$ with $|T| \leq p-5$. Since $p \geq 6$, there exists distinctvertices $x, y \in W$ such that $x, y \notin T$. If $x$ and $y$ are adjacent, then $x$ is non-adjacent to atleast one of $v_{i}$ and $v_{j}$, say $v_{j}$. Then $W_{1}=V\left(C_{p-1}\right)-\left\{x, v_{j}\right\}$ is a $s_{r}$ - set such that $W_{1} \neq W$ and $W_{1}$ properly contains $T$. If $x$ and $y$ are nonadjacent, then $W_{2}=V\left(C_{p-1}\right)-\{x, y\}$ isa $s_{r}$ - set such that $W_{2} \neq W$ and $W_{2}$ properly contains $T$. Thus $T$ is not a forcing subsetfor $W$. Now, we show that there exists a forcing subset of $W$ of cardinality $p-4$. Forconvenience, let $W=\left\{v_{2}, v_{4}, v_{5}, v_{6}, \ldots, v_{p-1}\right\}$. We show that $T_{1}=$ $\left\{v_{4}, v_{5}, v_{6}, \ldots, v_{p-1}\right\}$ is a forcing subset of $W$. If $T_{1}$ is not a forcing subset for $W$, then there exists as $s_{r}$-set $W^{\prime} \neq W$ such that $T_{1} \subseteq W^{\prime}$. Since $W^{\prime} \neq W,\left|W^{\prime}\right|=p-3$ and $\left|T_{1}\right|=p-$ $4, W^{\prime}$ must contain exactly one of $v_{1}$ or $v_{3}$. In any case, $\left\langle W^{\prime}\right\rangle$ is connected and so $W^{\prime}$ is not arestrained Steiner set of $G$, which is a contradiction. Hence $f_{r s}\left(W_{p}\right)=p-4$.

Theorem 2.16.If $W=\{u, v\}$ is a $s_{r}$ - set of a connected graph $G$, then $u$ and $v$ aretwo antipodal vertices of $G$.

Proof. Let $W=\{u, v\}$ be a $s_{r}$ - set of $G$. Then every vertex of $G$ lies on a Steiner $W$ - tree of $G$. Since every Steiner $W$ tree is a $u-v$ geodesic, every vertex of $G$ lies ona geodesic joining $u$ and $v$. Also the $\operatorname{subgraph} G[V-W]$ has no isolated vertices. Weclaim that $d(u, v)=d(G)$. If $d(u, v)<d(G)$, then let $x$ and $y$ be two vertices of $G$ suchthat $d(x, y)=d(G)$. Now, it follows that $x$ and $y$ lie on distinct geodesics joining $u$ and $v$. Hence 


\section{The Forcing Restrained Steiner Number of a Graph}

$d(u, v)=d(u, x)+d(x, v)(1)$

and

$d(u, v)=d(u, y)+d(y, v)$

(2)

By the triangle inequality,

$d(x, y) \leq d(x, u)+d(u, y)(3)$

Since $d(u, v)<d(x, y)$, (3) becomes

$d(u, v)<d(x, u)+d(u, y)$

(4)

Using (4) in (1), we get $d(x, v)<d(x, u)+d(u, y)-$ $d(u, x)=d(u, y)$. Thus,

$d(x, v)<d(u, y)$

Also, by triangle inequality, we have

$d(x, y) \leq d(x, v)+d(v, y)$

(6)

Now, using (5) and (2), (6) becomes $d(x, y)<d(u, y)+$ $d(v, y)=d(u, v)$. Thus, $d(G)<d(u, v)$ which is a contradiction. Hence $d(u, v)=d(G)$ so that $u$ and $v$ are antipodalvertices of $G$.

Theorem 2.17.If $G$ is a connected graph with $s_{r}(G)=2$, then $f_{r s}(G) \leq 1$.

Proof. Let $W=\{u, v\}$ be any $s_{r}$ - set of $G$. Then by Theorem 2.16, $u$ and $v$ are antipodalvertices of $G$. Suppose that $f_{r s}(G)=2$. Then it follows from Theorem 2.5 (c) that $W$ isnot the unique $s_{r}$ - set containing $u$ and so there exists $x \neq u$ in $G$ such that $W^{\prime}=\{u, x\}$ is also a $s_{r}$ - set of $G$. By Theorem 2.16, $u$ and $x$ are two antipodal vertices of $G$ and $v$ is an internal vertex of some $u-x$ geodesic in $G$. Therefore, $d(u, v)<d(u, x)$, which isa contradiction. Hence $f_{r s}(G) \leq 1$.

In view of Theorem 2.3, the following theorem gives a realization for the forcingrestrained Steiner number and the restrained Steiner number of a graph.

Theorem 2.18. For every pair $a, b$ of integers with $0 \leq a<$ $b, b \geq 2$, there exists aconnected graph $G$ such that $f_{r s}(G)=$ aand $s_{r}(G)=b$.

Proof. Suppose $a=0$. Let $G=K_{1, b-1}$. Then by Theorem 2.13, $f_{r s}(G)=0$ and fromTheorem $1.3, s_{r}(G)=b$. Now, assume that $a \geq 1$. For $b=a+1$, let $G=K_{1}+C_{a+3},(a \geq$ 1). By Theorem 2.15, $s_{r}(G)=a+1=b$ and $f_{r s}(G)=a$. For $b \neq a+1, \operatorname{let} F_{i}: r_{i}, s_{i}, t_{i}, u_{i}, v_{i}, r_{i}\left(1_{-} \leq i \leq a\right)$ be a copy of the cycle $C_{5}$. Let $G$ be the graph obtainedfrom $F_{i}(1 \leq i \leq a)$ by first identifying the vertices $v_{i-1}$ of $F_{i-1}$ and $t_{i}$ of $F_{i}(2 \leq i \leq a)$ and then adding $b-a$ new vertices $u, z_{1}, z_{2}, \ldots, z_{b-a-1}$ and joining the $b-a$ edges $u t_{1}$ and $v_{a} z_{i}(1 \leq i \leq b-a-1)$. The graph $G$ is given in Figure 2.4. Let $Z=\left\{u, z_{1}, z_{2}, \ldots, z_{b-a-1}\right\}$

be the set of all end-vertices of $G$. Let $H_{i}=\left\{r_{i}, s_{i}\right\}(1 \leq i \leq$ $a)$. First we show that $s_{r}(G)=b$. By Theorem $1.1, Z$ is a subset of every restrained Steiner set of $G$ and $\operatorname{sos}_{r}(G) \geq$ $b-a$. Since $S(Z) \neq V, Z$ is not a restrained Steiner set of $G$. We observethat every $S_{r}$ - set of $G$ must contain exactly one vertex from each $H_{i}(1 \leq i \leq a)$ and $\operatorname{sos}_{r}(G) \geq b-a+$ $a=b$. On the other hand, since the set $W=Z \cup$

$\left\{r_{1}, r_{2}, \ldots, r_{a}\right\}$ is aminimum restrained Steiner set of $G$, it follows that $s_{r}(G) \leq|W|=b$. Thus $s_{r}(G)=b$.

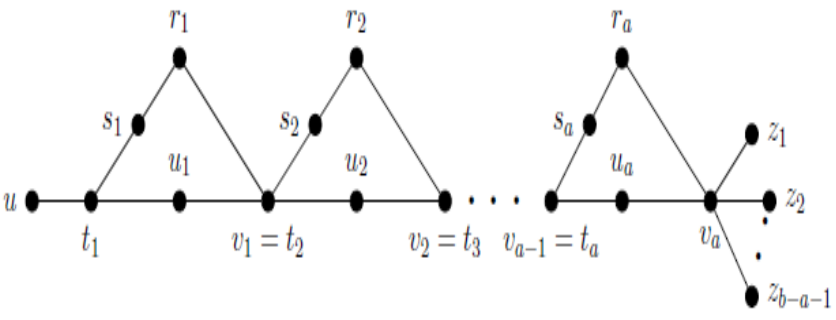

Figure 2.4

Next we show that $f_{r s}(G)=a$. Since every $s_{r}$ - set of $G$ contains $Z$, it follows fromTheorem 2.8 that $f_{r s}(G) \leq$ $s_{r}(G)-|Z|=b-(b-a)=a$. Now, since $s_{r}(G)=b$ and everyminimum restrained Steiner set of $G$ contains $Z$, it is easily seen that every minimumrestrained Steiner set $S$ is of the form $Z \cup\left\{c_{1}, c_{2}, \ldots, c_{a}\right\}$, where $c_{i} \in H_{i},(1 \leq i \leq a)$. Let $T$ be any proper subset of $S$ with $|T|<a$. Then there is a vertex $c_{j}(1 \leq i \leq a)$ such that $c_{j} \notin T$. Let $d_{j}$ be a vertex of $H_{j}$ distinct from $c_{j}$. Then $S_{1}=\left(S-\left\{c_{j}\right\}\right) \cup\left\{d_{j}\right\}$ is as ${ }_{r}^{-}$ setproperly containing $T$. Thus $S$ is not the unique $S_{r^{-}}$set containing $T$ and so $T$ is not aforcing subset of $S$. This is true for all $s_{r}$ - sets of $G$ and so it follows that $f_{r s}(G)=a$

\section{REFERENCES}

[1] F. Buckley and F. Harary, Distance in Graphs, Addition-Wesley, Redwood City, CA, 1990.

[2] G. Chartrand, P. Zhang, The Forcing Geodetic Number of a Graph, Discuss. Math. Graph Theory19(1999) $45-58$.

[3] G. Chartrand, P. Zhang, The Forcing Dimension of a graph, MathematicaBohemica, 126 (2001), $711-720$.

[4] G. Chartrand, P. Zhang, The Steiner number of a graph: a survey, Discrete Math., 242 (2002), 41-54.

[5] R. Eballe, S. Canoy Jr., Steiner sets in the join and composition of graphs, CongresusNumerantium, 170 (2004), $65-73$.

[6] F. Harary, Graph Theory, Addition-Wesley, 1969.

[7] C. Hernando, T. Jiang, M. Mora, I. M. Pelayo and C. Seara, On the Steiner, geodetic and hull number of graphs, Discrete Math. 293 (2005), 139-154.

[8] .J. John, M. S. Malchijah Raj, The Restrained Steiner Number of a Graph, (Communicated)

[9] M. S. MalchijahRaj,J. John, The Upper Restrained Steiner Number of a Graph, (Accepted)

[10] M. S. Malchijah Raj, J. John, The Restrained Edge Steiner Number of a Graph,Journal of Applied Science and Computations, 6 (2019), 1 - 8.

[11] I. M. Paleyo, Comment on "The Steiner number of a graph" by G. Chartrand and P. Zhang, Discrete Math. 242 (2002), 41 - 54.

[12] M. Raines, P. Zhang, The Steiner distance dimension of graphs, Australian J. Combin. 20 (1999), 133 143. 
[13] A. P. Santhakumaran and J. John, The Forcing Steiner number of a graph, DiscussionesMathematicae Graph Theory31 (2011), $171-181$.

[14]A. P. Santhakumaran and J. John, The Forcing Geodetic and the Forcing Steiner numbers of a graph, DiscussionesMathematicae Graph Theory31 (2011), $611-624$. 